\title{
Person Independent Facial Expression Detection using MBWM and Multiclass SVM
}

\author{
G.Nirmala Priya \\ Associate Professor, Department of ECE \\ Sona College of Technology, Salem
}

\author{
R.S.D.Wahida Banu, PhD. \\ Principal, Govt. College of Engineering \\ Salem
}

\begin{abstract}
Facial expression analysis is an attractive, challenging and important field of study in facial analysis. It's important applications include many areas such as human-computer interaction, human emotion analysis, biometric authentication, exhaustion detection and data-driven animation. For successful facial expression recognition, the first step is to arrive at an appropriate facial representation from original face image which is a crucial step. This paper, empirically evaluate facial representation using statistical features from the Local Binary Patterns, Simplified local binary mean and Mean based weight matrix for person-independent facial expression recognition. Multiclass SVM is applied systematically for classification. The Japanese female database JAFFE is used for the experiment. Extensive experiments shows that statistical features derived from LBP are effective and efficient for facial expression recognition. Further improved and best results are obtained with SLBM and MBWM features extracted using Multiclass Support Vector Machine classifiers.
\end{abstract}

\section{Keywords}

Face expression, LBP, SLBM, MBWM, Multiclass SVM

\section{INTRODUCTION}

The basic means of communication of the emotions and feeling of the human beings is by using Facial expression .Facial expression analysis is an attention-grabbing and demanding problem with impact on important applications in many areas including human-computer interaction and datadriven animation. Due to its wide range of applications, automatic facial expression recognition has attracted much attention in recent years. Much progress has been made in recognizing facial expression but, recognizing with high accuracy remains difficult due to the intricacy, complexity and variations of facial expressions. The first and the essential step for successful facial expression recognition is to deriving an effective facial representation from original face images. Some of the common approaches to extract facial features include geometric feature-based methods and appearance-based methods [1]. A geometric feature is the oldest method, which traces the shape and locations of facial components. These features are extracted to form a feature vector that represents the face geometry. Valstar et al. [2, 3] work on geometric feature-based method shows that geometric features provide a better performance than appearance-based approaches in face expression recognition. But, the geometric feature-based methods are difficult to estimate in many situations.

In appearance-based methods, rather than processing the spatial information, the pixel information are processed using filters, such as Gabor wavelets, Haar wavelet are applied to the whole-face or specific face-regions to extract the appearance changes of the face. Since, appearance- based method have superior performance, more works are focused on this methods $[4,5]$. Still, it is intensive both in time and memory to convolve face images with a bank of Gabor filters to extract multi-scale and multi-orientation coefficients. This paper empirically study facial representation based on Local Binary Pattern (LBP), Simplified Local Binary Mean (SLBM) and Mean Based Weight Matrix (MBWM) features [6-9] for person-independent facial expression recognition.LBP features were proposed originally for texture analysis, and recently have been introduced to represent faces in facial images analysis [10-12]. The most important properties of LBP features are their tolerance against illumination changes and their computational simplicity. The SLBM features are used for face detection which gives a better performance compared to the LBP features. The machine learning method, Support Vector Machine (SVM) and Multiclass SVM technique are studied and the multiclass SVM is performed on facial expression recognition using LBP, SLBM and MBWM features.

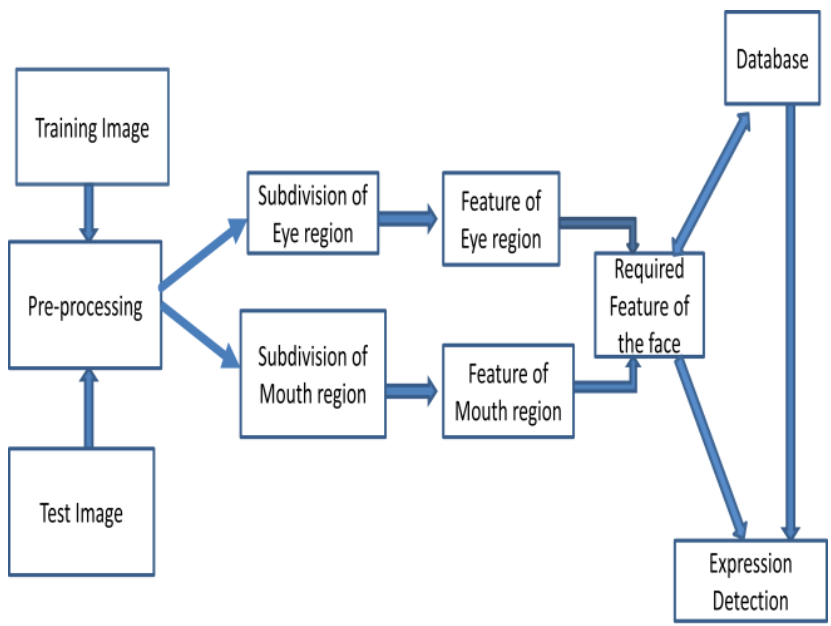

Figure 1. General Block Diagram

LBP features can be derived very fast in a single scan through the raw image and lie in low-dimensional feature space, and it still retaining discriminative facial information in a compact representation. The limitation of the existing facial expression recognition methods is that they attempt to recognize facial expressions from data collected in a highly controlled environment given high resolution frontal faces [13]. But, in real world applications low resolution images are only available, which will be much more difficult to work with. In [13], Tian studied the effects of different image resolutions for each step of automatic facial expression recognition. This 
paper, investigate the various features for images including low-resolution facial images. Experiments on low resolution images show that LBP features perform stably and robustly over a useful range of low resolutions of face images. This encouraging performance on low resolution images illustrated the promising and better performance of the MBWM features for low-resolution images. Figure.1 presents the basic block diagram of the facial expression recognition system.

\section{RELATED WORKS}

Facial expression recognition has attracted the attention of the persons involved in behavioral studies .Suwa et al. [16] made the first attempt to automatically analyze facial expressions from image sequences. Much progress has been made in the last decade, and a thorough survey of the exiting work can be found in $[14,15]$. The two important steps of Facial expression recognition are: facial feature extraction and design of classifier. Facial features are derived from original face images to effectively represent faces. The 1 features that are extracted should minimize within-class variations of expressions and maximize the between class variations. If the features are inadequate, even the best classifier could fail to achieve accurate recognition. In some cases, Facial geometry has been widely used in facial representation [18].In this method shapes and locations of facial components are extracted to represent the face geometry. Zhang et al. [17]in his analysis used the geometric positions of 34 fiducial points as facial features to represent face image. Valstar et al. [2] in his paper analyzed Action Unit(AU) detection by classifying features that are calculated from tracked fiducial facial points. This method classifies the expressions with higher recognition rate. From this, it is clear that the facial representation based on tracked facial points is well suited for facial expression analysis. Recently Valstar et al. [3] further analyzed in his paper with a fully automatic AU detection system that can automatically localize facial points in the first frame and recognize AU segments using a subset selected by AdaBoost. Yet, the geometric feature-based representation normally requires accurate and reliable facial feature detection, which is difficult to achieve in many situations.[21] To exploit the temporal behaviors of facial expressions, different techniques were presented for facial expression recognition in image sequences. There have been several attempts to track and recognize facial expressions over time based on optical flow analysis [5,6]. Tian et al. [10] presented a Neural Network based approach to recognize facial action units in image sequences. Hidden Markov Models (HMMs) have been widely used to model the temporal behaviors of facial expressions from image sequences [11,13]. Cohen et al. [11] proposed a multi-level HMM classifier. This allows facial expression classification on a video segment, and also to automatically segment a long video sequence to the different expressions segments without resorting to heuristic methods of segmentation. But HMMs cannot deal with dependencies in observation. Dynamic Bayesian Networks (DBNs) recently were exploited for sequence-based expression recognition [16, 14,18].Zhanget.al[19],measured facial features simultaneously by using an active Infra-Red illumination and Kalman filtering to deal with large variations of head motion. The other method to represent faces is to model the appearance changes of faces. Spatial analysis method including Principal Component Analysis (PCA), Linear Discriminant Analysis (LDA) and Independent Component Analysis (ICA) and Gabor wavelet analysis can be applied to either the wholeface or specific face regions to extract the facial appearance changes. Better performances were obtained by using Gaborwavelet representation and ICA. Due to their superior performance, Gabor-wavelet representations have been widely adopted in face image analysis [20]. But, the computation of Gabor-wavelet representations is both time and memory for face image has the high dimensionality. in recent times, Local Binary Patterns have been introduced as effective appearance features for facial image analysis [10-12]. Liao et.al [21] compared LBP features with Gabor features for facial expression recognition, and studied their performance over a range of image resolutions. Liao et.al also included the facial expression manifold learning using LBP feature space. More recently, Liao et al. [22] introduced an improved LBP operator to extract features in both intensity and gradient maps for facial expression recognition, and also tested their methods on facial images of reduced resolutions. However, their experiment was carried out on a very small database (213 images from 10 subjects). In this paper, we compare LBP, SLBM and MBWM features for facial expression recognition using JAFFE databases.

\section{FACIAL EXPRESSION DATA}

Facial expressions include different emotions of different persons. A Popularly used facial expression description is Facial Action Coding System (FACS).This is a humanobserver-based system developed to capture fragile changes in facial expressions. In FAC system, facial expressions are decomposed into one or more Action Units (AUs). This unit is more popularly used and has grabbed more attention. Psychophysical studies point out that basic emotions have corresponding universal facial expressions across all cultures. So, the present facial expression recognition systems attempt to recognize a set of prototypic emotional expressions that includes disgust, fear, joy, surprise, sadness and anger. This paper also focuses on the same prototypic expression recognition. We consider both 6-class and 7-class expression recognition by including the neutral expression. The experiment was conducted on the Japanese female database JAFFE, one of the most comprehensive databases in the current facial-expression-research community. Figure. 2 shows some sample images from the Japanese female database. The database was planned and assembled by Miyuki Kamachi, Michael Lyons, 

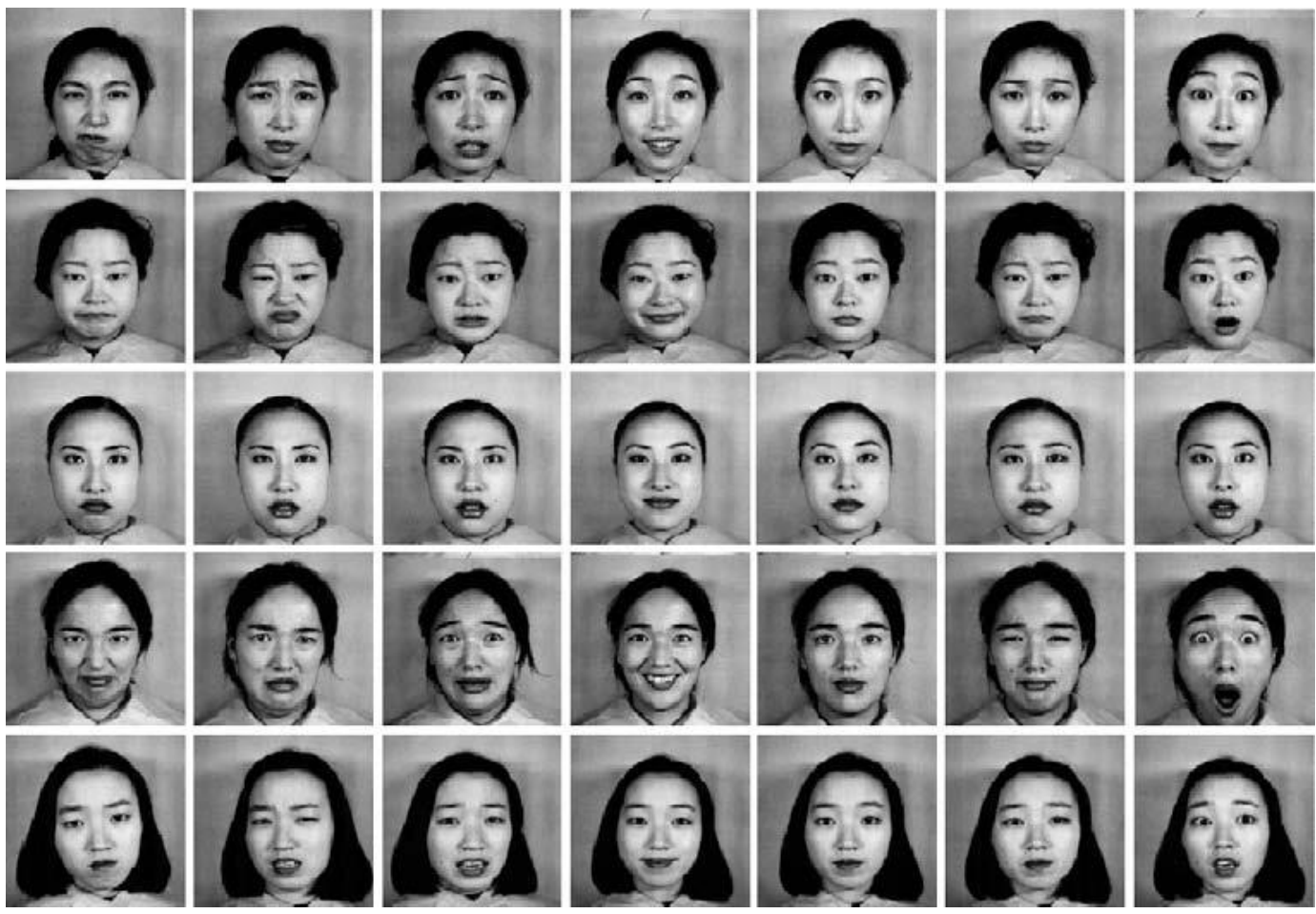

Figure 2 Sample Images of JAFFE database

and Jiro Gyoba with the help of Reiko Kubota as a research assistant. The photos were taken at the Psychology Department in Kyushu University. The JAFFE database used in this study contains 213 images of female facial expressions. Each image has a resolution of 256X256 pixels. The number of images corresponding to each of the 7 categories of expression (neutral, happiness, sadness, surprise, anger, disgust and fear) is almost the same. The images in the database are grayscale images in the tiff file format. The expression of each person along with a semantic rating is provided in the database. This makes the database suitable for facial expression research. Most of the images in the database are frontal image. Original images have already been rescaled and cropped such that the eyes are roughly at the same position with a distance of 60 pixels in the final images.

The face images are referred with their initials as KA, KL, KM, KR, MK, NA, NM, TM, UY and YM. Each image in the database was rated by 91 experimental subjects for degree of each of the six basic expressions present in the image. The semantic rating of the images showed that the error for the fear expression was higher than that for any other expression but there exist a number of cases even for other expressions in which the expression getting highest semantic rating is different from the expression label of the image. More precisely, the dataset is partitioned randomly into ten groups of roughly equal numbers of subjects. Nine groups were used as the training data to train classifiers, while the remaining group was used as the test data. The above process was repeated ten times for each group in turn to be omitted from the training process.

The average recognition results on the test sets are being considered as recognition rate. Facial images of 110 X 150 pixels were cropped from original frames based on the two eyes location. Since the expression variation is restricted to the eye and the mouth region the eye region and the mouth region are located and the LBP, SLBM and MBWM features are estimated. No further preprocessing steps such as alignments of mouth were performed in this algorithm. Since, the faces in the database are frontal view, head pose changes are not considered. For realistic sequences with head pose variation, head pose estimation can be adopted to detect front or near front view. Illumination changes exist in the database, but the illumination changes are not removed. Figure. 3 shows an example of the original face image and the cropped image. Figure 4 represents the 7 expressions for a subject and the extraction of the eyes and the mouth region which shows the variation in expression

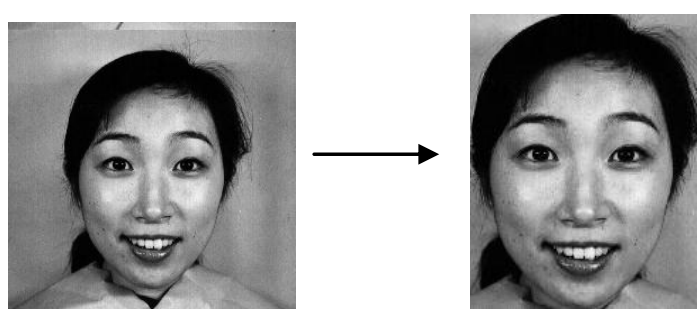

Figure 3. Sample of Original and cropped image

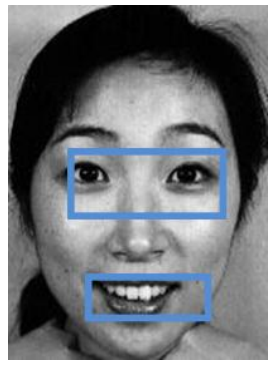

Joy

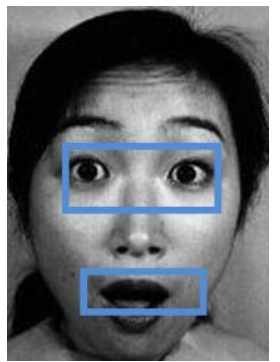

Surprise 


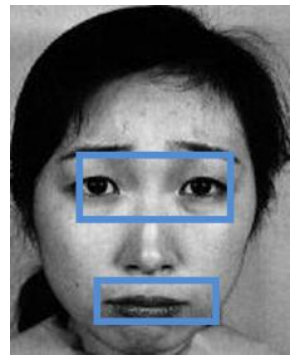

Sad

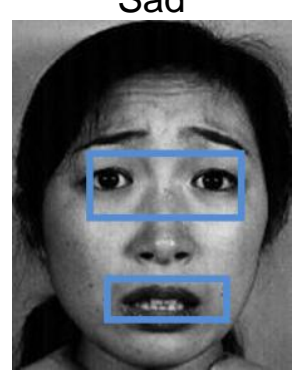

Fear
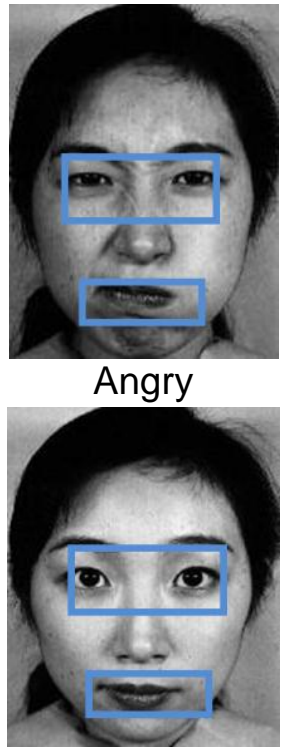

Neutral

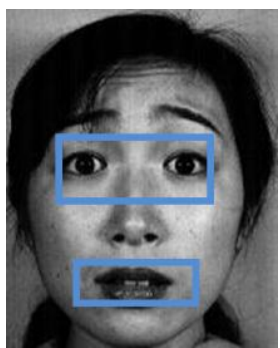

Disgust

Figure 4. Region subdivision for the 7 expressions

\section{FEATURE EXTRACTION}

\subsection{Local Binary Pattern (LBP)}

LBP operator was originally designed for texture description and was proved a powerful means of texture description. The operator labels the pixels of an image by thresholding a 3 X3 neighborhood of each pixel with the center value and considering the results as a binary number Figure.5 shows the, extraction of Local Binary Pattern (LBPs). It has also shown a good performance in the area of facial image description. The derived binary numbers (called Local Binary Patterns or LBP codes) represents the local primitives including different types of curved edges, spots, flat areas, etc .so each LBP code can be regarded as a micro-texton. The basic LBP operator labels the pixels of an image (Ip) by thresholding each $3 \times 3$ pixel neighborhood of the input image with the center pixel value (Ic), multiplying the threshold values by weight (powers of two) and summing them. Thresholding is done using the centre pixel as in Eq. 1:

$f(I p-I c)=\left\{\begin{array}{c}1, I_{p} \geq I_{c} \\ 0, \text { otherwise }\end{array}\right.$

\begin{tabular}{|l|l|l|}
\hline 110 & 154 & 160 \\
\hline 150 & 150 & 69 \\
\hline 142 & 105 & 152 \\
\hline
\end{tabular}$\rightarrow$\begin{tabular}{|l|l|l|}
\hline $\mathbf{0}$ & $\mathbf{1}$ & $\mathbf{1}$ \\
\hline $\mathbf{1}$ & & $\mathbf{0}$ \\
\hline $\mathbf{0}$ & $\mathbf{0}$ & $\mathbf{1}$ \\
\hline
\end{tabular}

Figure 5. LBP operator

Weights are then assigned and the LBP values are obtained using Eq. 2 and the values are summed to obtain the LBP values for the $3 \square 3$ matrix. The LBP feature thus obtained are considered for classification purpose:

$L B P=\sum_{p=0}^{7} f(I p-I c) 2^{p}$

LBPs have been very effective for image representation as it is being applied to visual inspection, motion detection and outdoor scene analysis. The most important properties of LBP features are their tolerance against monotonic illumination changes and their computational simplicity. The LBP operator mainly detects many texture primitives as spot, line end, edge

and corner typically accumulated into a histogram over a region to capture local texture information. The statistical features as mean and standard deviation of the LBP features extracted are used for classification.

\subsection{Simplified Local Binary Mean (SLBM)}

The simplified local binary mean overcomes the disadvantage of the LBP algorithm by using the mean of the 9 pixels for thresholding. This method involves three steps which include subdividing, thresholding and weighing. First, a $3 \square 3$ sub image is cropped [8].The pixel values are represented as Ip. Thresholding is done using the mean of the 9 elements of the $3 \square 3$ sub image (Im). Thresholding is done based on the rule given in Eq. 3:

$f(I p-I m)=\left\{\begin{array}{c}1, I_{p} \geq I_{m} \\ 0, \text { otherwise }\end{array}\right.$

Weights are then assigned and summed to obtain the SLMB values for the $3 \square 3$ matrix using Eq. 4 . The

$S L B M=\sum_{p=0}^{7} f(I p-I m) 2^{p}$

SLMB feature thus obtained are considered for classification purpose:

The SLMB features are thus calculated. Many images of different types can have similar histograms. Histograms provide only a coarse characterization of an image. This is the main disadvantage of using histograms. So, the statistical features such as mean and standard deviation of the SLMB features are calculated.

\subsection{Mean Based Weight Matrix (MBWM)}

In LBP, thresholding is exactly at the value of the central pixel ic. This makes it to be sensitive to noise, especially in near-uniform image regions. Many facial regions are relatively uniform. It is potentially useful to improve the robustness of the underlying descriptors in these areas. Also, in SLBM only two values are assigned and the weightage is not given to the mean value. So, the SLBM is extended to Mean Based Weight Matrix (MBWM) [9].The mean based weight matrix involves three steps which include subdividing, 
thresholding and weighing. The $3 \square 3$ pixels of the image are replaced by a 3-valued function as given in Eq. 5:

$f(I p-I m)=\left\{\begin{array}{c}2, \quad I_{p}>I_{m} \\ 1, \quad I_{p}=I_{m} \\ 0, \text { Otherwise }\end{array}\right.$

Weights are then assigned and summed to obtain the

MBWM values as in Eq. 6 for the $3 \square 3$ matrixes:

$M B W M=\sum_{p=0}^{7} f(I p-\operatorname{Im}) 2^{p}$

The MBWM features are thus calculated. The first order statistical features such as mean and standard deviation of the MBWM features are calculated. These features are used for classification. Initially, the eye and the mouth region are located in the image and the statistical features are extracted. Again the statistical features are extracted for the remaining portion of the image . The total features are considered by giving more weightage to the mouth and then to eye and then to the remaining portion of the image. This gives the added advantage to this method.

\section{FACE EXPRESSION RECOGNITION}

\subsection{Support Vector Machine (SVM)}

As a powerful machine learning technique for data classification, SVM performs an implicit mapping of data into a higher (maybe infinite) dimensional feature space and then finds a linear separating hyperplane with the maximal margin to separate data in this higher dimensional space[8]. Given a training set of labeled examples $\{(x i, y i), i=1,2 \ldots \ldots .1\}$ where $\mathrm{xi} \in \mathrm{Rn}$ and yi $€\{-1,1\}$ a new test example $\mathrm{x}$ is classified by the function as in Eq. 7:

$f(x)=\operatorname{sgn}\left(\sum_{i=1}^{l} \alpha_{i} y_{i} K\left(x_{i}, x\right)+b\right)$

where, $\alpha \mathrm{i}$ is the Lagrange multiplier of a dual optimization problem that describes the separating hyperplane $\mathrm{K}(\mathrm{xi}, \mathrm{x})$ is a kernel function and $b$ is the threshold parameter of the hyperplane. The training sample xi with $\alpha \mathrm{i}>0$ is called support vectors and SVM finds the hyperplane that maximizes the distance between the support vectors and the hyperplane. Given a non-linear mapping $\Phi$ that embeds the input data into the high dimensional space, kernels have the form of

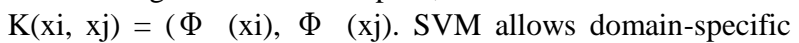
selection of the kernel function. Though new kernels are being proposed, the most frequently used kernel functions are the linear, polynomial and Radial Basis Function (RBF) kernels. SVM makes binary decisions. With regard to the parameter selection of SVM, the mean and standard deviation are chosen. These parameters provided the best accuracy. The generalization performances achieved using the two different kernels is discussed.SVM makes binary decisions, so the multi-class classification is accomplished here, by using the one-against-rest technique, which trains binary classifiers to discriminate one expression from all others, and outputs the class with the largest output of binary classification.

\section{EXPERIMENTS}

In this section, a person-independent facial expression recognition using LBP, SLBM and MBWM features are analyzed. Multiclass Support Vector Machines is used to examine the various facial expressions. The confusion matrices of 7-class recognition with the RBF kernel are shown in Tables 1, 2 and 3. It is observed that, Joy, Surprise and
Neutral can be recognized with high accuracy (90-98\%), while the recognition rates for Fear and Sadness are much lower (79-88\%). the recognition performance for almost all the expression is increased. The performances comparison of the linear and RBF kernel is stated in Table 4. Comparing the performance for the 6-class problem and 7-class problem, the accuracy of classification is increased in 6 class problem compared to the classification accuracy of 7 class classifier.

Table 1 Confusion matrix of 7-class facial expression

Recognition for LBP features using RBF SVM

\begin{tabular}{|c|c|c|c|c|c|c|c|}
\hline & Anger & Disgust & Fear & Joy & Sad & Surprise & Neutral \\
\hline Anger & $\mathbf{8 6 . 8}$ & 3.7 & 2.3 & 0 & 3.2 & 0 & 3.7 \\
\hline Disgust & 1.8 & $\mathbf{7 8 . 8}$ & 0 & 0 & 14.08 & 0 & 5.16 \\
\hline Fear & 0 & 4.2 & $\mathbf{8 8 . 7}$ & 0 & 5.16 & 0 & 1.8 \\
\hline Joy & 0 & 0 & 4.69 & $\mathbf{9 4 . 3 1}$ & 0 & 3.28 & 3.7 \\
\hline Sad & 0 & 6.1 & 6.57 & 0 & $\mathbf{7 9 . 8}$ & 0.09 & 7.04 \\
\hline Surprise & 0 & 2.3 & 0 & 0 & 0.09 & $\mathbf{9 8 . 1 2}$ & 0 \\
\hline Neutral & 0 & 3.7 & 2.8 & 0 & 3.2 & 0 & $\mathbf{9 0 . 1 4}$ \\
\hline
\end{tabular}

Table 2 Confusion matrix of 7-class facial expression Recognition for SLBM features using RBF SVM

\begin{tabular}{|c|c|c|c|c|c|c|c|}
\hline & Anger & Disgust & Fear & Joy & Sad & Surprise & Neutral \\
\hline Anger & $\mathbf{8 9 . 2}$ & 2.8 & 18 & 0 & 2.8 & 0 & 3.2 \\
\hline Disgust & 1.4 & $\mathbf{8 0 . 7 5}$ & 0 & 0 & 13.14 & 0 & 4.69 \\
\hline Fear & 0 & 4.69 & $\mathbf{8 9 . 6 7}$ & 0 & 4.69 & 0 & 0.09 \\
\hline Joy & 0 & 0 & 3.28 & $\mathbf{9 4 . 8 3}$ & 0 & 2.8 & 3.7 \\
\hline Sad & 0 & 5.6 & 6.1 & 0 & $\mathbf{8 4 . 0 3}$ & 0 & 5.16 \\
\hline Surprise & 0 & 0 & 0 & 1.87 & 0 & $\mathbf{9 6 . 7}$ & 0 \\
\hline Neutral & 0 & 3.7 & 2.3 & 0 & 2.8 & 0 & $\mathbf{9 1 . 0 7}$ \\
\hline
\end{tabular}

Table 3 Confusion matrix of 7-class facial expression Recognition for MBWM features using RBF SVM

\begin{tabular}{|c|c|c|c|c|c|c|c|}
\hline & Anger & Disgust & Fear & Joy & Sad & Surprise & Neutral \\
\hline Anger & $\mathbf{9 2 . 4 8}$ & 3.75 & 0 & 0 & 1.4 & 0 & 2.3 \\
\hline Disgust & 0 & $\mathbf{8 3 . 5 6}$ & 0 & 0 & 12.2 & 0 & 4.2 \\
\hline Fear & 0 & 4.2 & $\mathbf{9 1 . 0 7}$ & 0 & 3.75 & 0 & 0.09 \\
\hline Joy & 0 & 0 & 0 & $\mathbf{9 5 . 4}$ & 0 & 1.8 & 2.8 \\
\hline Sad & 0 & 4.2 & 6.10 & 0 & $\mathbf{8 5 . 4 4}$ & 0 & 4.2 \\
\hline Surprise & 0 & 0.09 & 0 & 0.09 & 0 & $\mathbf{9 8 . 1 2}$ & 0 \\
\hline Neutral & 0 & 3.75 & 0.09 & 0 & 2.3 & 0 & $\mathbf{9 2 . 9 5}$ \\
\hline
\end{tabular}

This is presented in table 5. Table 6 shows a comparison of 6 class expression and 7 class expression. The LBP features are compared with the SLBM and MBWM features for facial expression recognition using SVMs. SVMs with linear and RBF kernels were used to classify 7-class expressions. RBF kernels performed better than the linear kernel.Figure.6 shows that the 6 class classifier performance is better compared to 7 class classifier. 


\section{Performance Analysis}

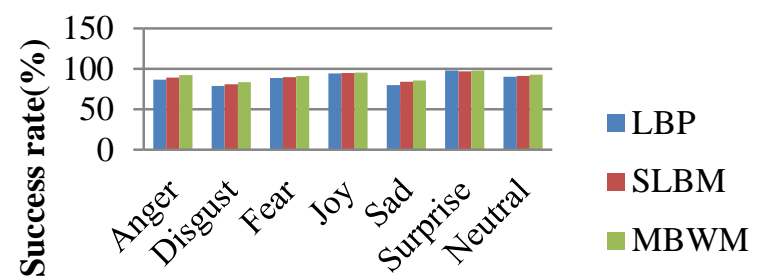

Facial Expressions

Figure 6.Performance Analysis of SVM (RBF) Classifier

Table 4 Comparison of Linear and RBF SVM

\begin{tabular}{|c|c|c|}
\hline & Linear SVM & RBF SVM \\
\hline LBP & 85.62 & 88.09 \\
\hline SLBM & 88.05 & 89.46 \\
\hline MBWM & 89.42 & 91.35 \\
\hline
\end{tabular}

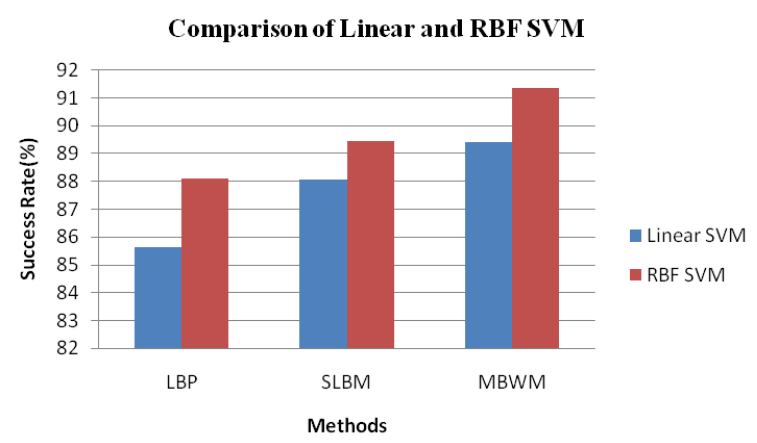

Figure 7.Performance Analysis of Linear and RBF Classifier

Table 5 Confusion matrix of 6-class facial expression recognition using MBWM features using RBF SVM

\begin{tabular}{|c|c|c|c|c|c|c|}
\hline & Anger & Disgust & Fear & Joy & Sad & Surprise \\
\hline Anger & $\mathbf{9 3 . 8 9}$ & 3.75 & 0.09 & 0 & 1.4 & 0 \\
\hline Disgust & 0 & $\mathbf{8 6 . 3 8}$ & 0 & 13.6 & 0 & 0 \\
\hline Fear & 0 & 4.69 & $\mathbf{9 1 . 0 7}$ & 0. & 3.75 & 0 \\
\hline Joy & 0 & 0 & 2.8 & $\mathbf{9 5 . 4}$ & 0 & 1.8 \\
\hline Sad & 0 & 4.2 & 6.1 & 1.4 & $\mathbf{8 8 . 2 6}$ & 0 \\
\hline Surprise & 0 & 0.09 & 0 & 0.09 & 0 & $\mathbf{9 8 . 1 2}$ \\
\hline
\end{tabular}

Comparision of 6 expressions using MBWM features

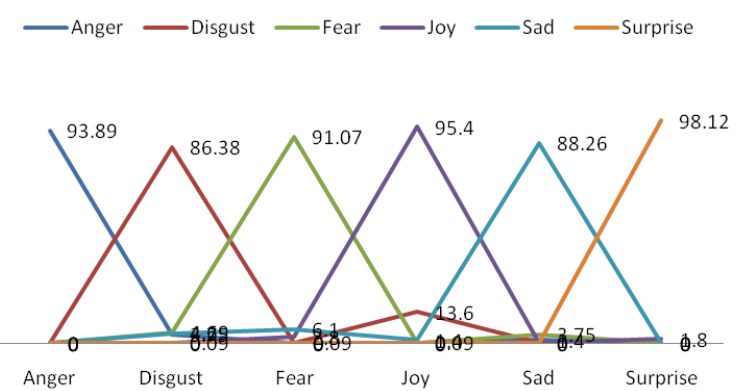

Figure 8.Performance Analysis for 6 expressions using RBF SVM Classifier

Table 6 Performance of 6 class and 7 class classifier

\begin{tabular}{|c|c|c|c|c|}
\hline & \multicolumn{2}{|c|}{ Linear SVM } & \multicolumn{2}{c|}{ RBF SVM } \\
\hline & $\begin{array}{c}\text { 6 class } \\
\text { recognit } \\
\text { ion }(\%)\end{array}$ & $\begin{array}{c}7 \text { class } \\
\text { recognitio } \\
\mathrm{n}(\%)\end{array}$ & $\begin{array}{c}\text { 6 class } \\
\text { recognitio } \\
\mathrm{n}(\%)\end{array}$ & $\begin{array}{c}7 \text { class } \\
\text { recognitio } \\
\mathrm{n}(\%)\end{array}$ \\
\hline LBP & 87.52 & 85.62 & 89.52 & 88.09 \\
\hline SLBM & 89.02 & 88.05 & 90.82 & 89.46 \\
\hline MBWM & 90.14 & 89.42 & 92.18 & 91.35 \\
\hline
\end{tabular}

6 class Vs 7 class Expression

$\because \mathrm{LBP} \backsim \mathrm{SLBM} \cong \mathrm{MBWM}$

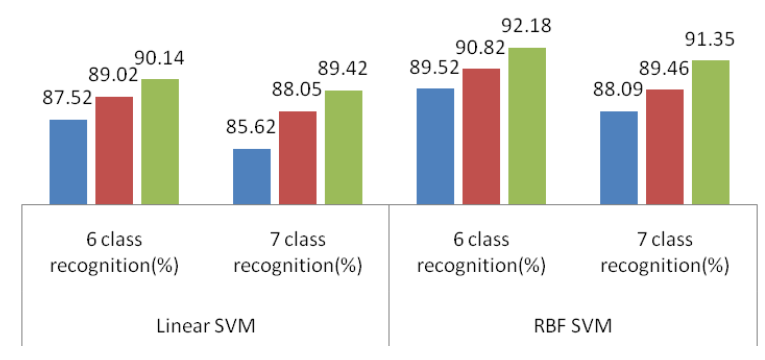

Figure 9.Performance of 6 class and 7 class classifier

The MBWM features give better features for the RBF SVM classifier.Figure. 7 shows that the performance of RBF classifier is better compared to linear SVM classifier. The 6 expression class performance using RBF SVM is elaborated in figure.8.A detailed performance analysis of the 3 different feature extraction algorithm with the 2 SVM classifier are detailed in figure.9.

\section{CONCLUSION}

In this paper, an efficient facial expression representation and classification methodology is proposed. Facial Expression representation are based on LBP,SLBM and MBWM features .These features are classified using the multiclass support vector machine. The seven universal expressions i.e. anger, disgust, fear, happy, sad, Neutral and surprise are recognized with $100 \%$ accuracy for one subject's images and as high as $91.35 \%$ accuracy with the combination of 10 subject's images using all the 7 classes and $92.18 \%$ using 6 classes of expressions. 


\section{ACKNOWLEDGEMENT}

We would like to thank Dr. Michael J. Lyons for the use of the JAFFE database.

\section{REFERENCES}

[1] Y. Tian, T. Kanade, J. Cohn, Handbook of Face Recognition, Springer, 2005 (Chapter 11. Facial Expression Analysis).

[2] M. Valstar, I. Patras, M. Pantic, Facial action unit detection using probabilistic actively learned support vector machines on tracked facial point data, in: IEEE Conference on Computer Vision and Pattern Recognition Workshop, vol. 3, 2005, pp. 76-84.

[3] M. Valstar, M. Pantic, Fully automatic facial action unit detection and temporal analysis, in: IEEE Conference on Computer Vision and Pattern Recognition Workshop, 2006, p. 149.

[4] [4] M.J. Lyons, J. Budynek, S. Akamatsu, Automatic classification of single facial images, IEEE Transactions on Pattern Analysis and Machine Intelligence 21(12) (1999) 1357-1362.

[5] G. Donato, M. Bartlett, J. Hager, P. Ekman, T. Sejnowski, Classifying facial actions, IEEE Transactions on Pattern Analysis and Machine Intelligence 21(10) (1999) 974-989.

[6] T. Ojala, M. Pietikäinen, D. Harwood, A comparative study of texture measures with classification based on featured distribution, Pattern Recognition 29 (1) (1996) 51-59.

[7] T. Ojala, M. Pietikäinen, T. Mäenpää, Multiresolution gray-scale and rotation invariant texture classification with local binary patterns, IEEE Transactions on Pattern Analysis and Machine Intelligence 24 (7) (2002) 971987.

[8] Priya, G.N. and R.S.D.W. Banu, 2012. A Simplified Local Binary Mean (SLBM) based human gender classification. Eur. J. Sci. Res., 71: 435-442.

[9] Priya, G.N. and R.S.D.W. Banu, 2012. Detection of Occluded Face Image using Mean Based Weight Matrix and Support Vector Machine, J. Computer Sci., 8 (7): 1184-1190

[10] T. Ahonen, A. Hadid, M. Pietikäinen, Face recognition with local binary patterns, in: European Conference on Computer Vision (ECCV), 2004.

[11] A. Hadid, M. Pietikäinen, T. Ahonen, A discriminative feature space for detecting and recognizing faces, in: IEEE Conference on Computer Vision and Pattern Recognition (CVPR), 2004.
[12] X. Feng, A. Hadid, M. Pietikäinen, A coarse-to-fine classification scheme for facial expression recognition, International Conference on Image Analysis and Recognition (ICIAR), Lecture Notes in Computer Science, vol. 3212, Springer, 2004, pp. 668-675.

[13] Y. Tian, Evaluation of face resolution for expression analysis, in: CVPR Workshop on Face Processing in Video, 2004.

[14] M. Pantic, L. Rothkrantz, Automatic analysis of facial expressions: the state of art, IEEE Transactions on Pattern Analysis and Machine Intelligence 22 (12)(2000) 1424-1445.

[15] B. Fasel, J. Luettin, Automatic facial expression analysis: a survey, Pattern Recognition 36 (2003) 259-275.

[16] M. Suwa, N. Sugie, K. Fujimora, A preliminary note on pattern recognition of human emotional expression, in: International Joint Conference on Pattern Recognition, 1978, pp. 408-410.

[17] Z. Zhang, M.J. Lyons, M. Schuster, S. Akamatsu, Comparison between geometry-based and Gaborwavelets-based facial expression recognition using multilayer perceptron, in: IEEE International Conference on Automatic Face \& Gesture Recognition (FG), 1998.

[18] M. Pantic, I. Patras, Dynamics of facial expression: recognition of facial actions and their temporal segments from face profile image sequences, IEEE Transactions on Systems, Man, and Cybernetics 36 (2) (2006) 433-449.

[19] Y. Zhang, Q. Ji, Active and dynamic information fusion for facial expression understanding from image sequences, IEEE Transactions on Pattern Analysis and Machine Intelligence 27 (5) (2005) 1-16.

[20] M.S. Bartlett, G. Littlewort, M. Frank, C. Lainscsek, I. Fasel, J. Movellan, Recognizing facial expression: machine learning and application to spontaneous behavior, in: IEEE Conference on Computer Vision and Pattern Recognition (CVPR), 2005.

[21] C. Shan, S. Gong, P.W. McOwan, Robust facial expression recognition using local binary patterns, in IEEE International Conference on Image Processing(ICIP), Genoa, vol. 2, 2005, pp. 370-373.

[22] S. Liao, W. Fan, C.S. Chung, D.-Y. Yeung, Facial expression recognition using advanced local binary patterns, tsallis entropies and global appearance features, in: IEEE International Conference on Image Processing (ICIP), 2006,pp. 665-668. 